\title{
SIMULATION OF YARD OPERATIONS AND MANAGEMENT IN TRANSSHIPMENT TERMINALS
}

\author{
Uwe Clausen \\ Ina Goedicke \\ TU Dortmund University \\ Institute of Transport Logistics \\ Leonhard Euler Str. 2 \\ D-44227 Dortmund, GERMANY
}

\begin{abstract}
Due to intensified globalization of supply networks and growing e-commerce activities, logistics service providers have to deal with steadily increasing shipment volumes. Highly performing transshipment terminals have been identified as an essential basis to handle those volumes within transportation networks. In recent years, internal sorting processes have already been the focus of analysis, standardization and optimization. In contrast to that, yard management in the terminals is still operated with very limited automated intelligence. Due to the fact that performance of internal sorting operations can only be achieved by constantly high input flows, an enhanced efficiency of yard operations is the main challenge to increase the performance of transshipment terminals. Therefore a simulation method for yard operations in terminals has been developed which allows detailed analysis. Furthermore, it has been applied on an exemplary terminal and different controlling strategies have been tested concerning their impact on performance aspects.
\end{abstract}

\section{INTRODUCTION}

Intensified globalization of supply networks and growing e-commerce activities lead to steadily growing shipment volumes for logistics service providers. Additionally, service requirements for business customers such as late pickups and early deliveries get more and more important also for non-express transportation networks. To stay competitive, service providers have to measure the performance of all processes within the transportation chain and identify optimization potential within their operations. This is done also with regard to improved ecological efficiency which is required from political instances as well as from customers.

The service providers operate on a network of different depots, each covering a specific geographical region. The network is open for nearly all types of customers, no long-term contracts have to be made. Until late afternoon shipments are collected at customers and brought to the regional depot (outbound shift). Afterwards, the shipments get sorted by destinations and transported to the depot located in the region of the consignee (line haul transport). Shipments are sorted there according to delivery tours which take place in the morning (inbound shift). Consequently, the operations of a service provider can be divided into mobile transportation processes on the one hand and stationary transshipment processes in the depots on the other hand. The upper part of figure 1 demonstrates the sequence of transportation processes. Terminal operations are indicated in the lower part. Black data curves in between are demonstrating a typical arrival pattern of shipment volumes in the terminal. Since the time slots for transportation are fixed due to geographic reasons and customer demands the quality of the whole network largely depends on the reliability in meeting cut-off times in the network nodes. The first challenge are increasing ship- 


\section{Clausen and Goedicke}

ment volumes which have to be handled in the terminals. Secondly, arrival times postpone due to late pickups and increased transportation times. Transportation times are prolonged by the consolidation of transportation processes that results in additional distances and handling processes (integration of hub or increased utilization of trucks by more stops per tour). Although network planning tries to consider the requirement of an equalized system load profile for the receiving depots to certain extent the main focus is the consolidation of shipments and transportation units due to high costs for transportation. The impact of those effects on incoming shipment volumes is indicated by the dashed black data curve.

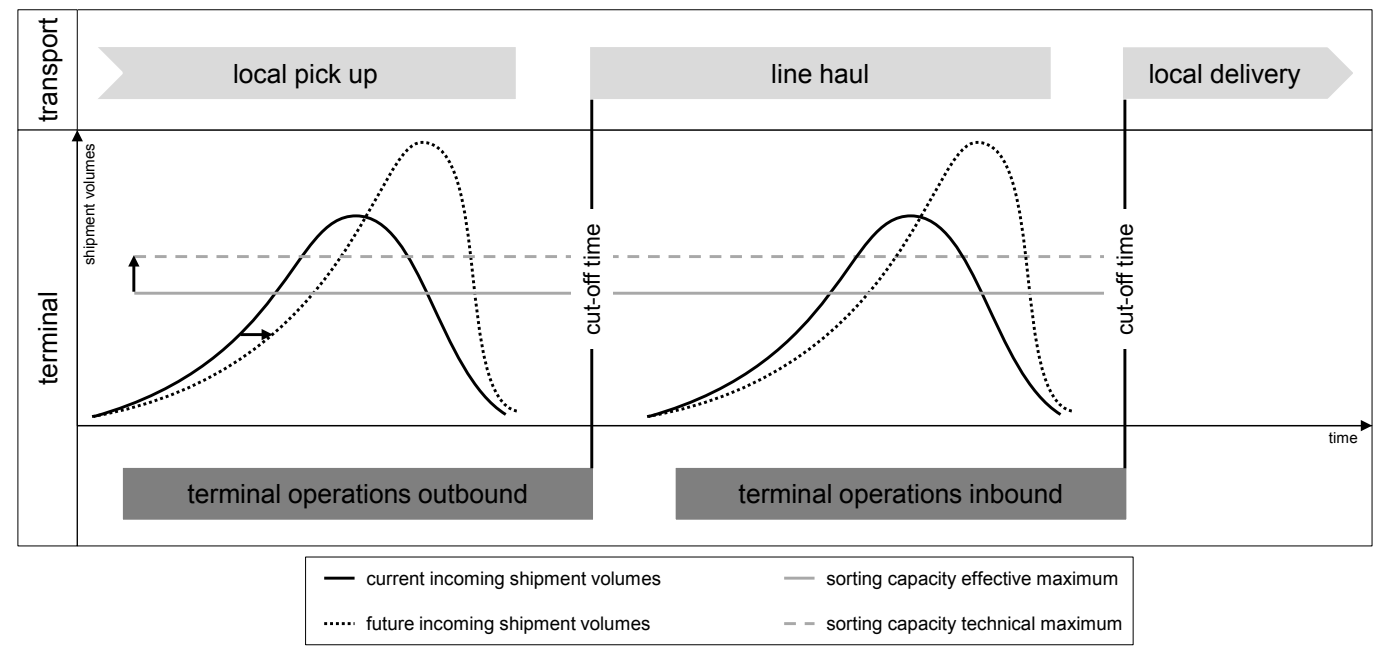

Figure 1: Interdependencies between transportation and terminal operations

Concluding, highly performing network nodes are the essential basis for competitive networks of logistics service providers. In recent years, internal sorting processes have already been the focus of analysis, standardization and optimization. In contrast to that, yard management within the network nodes especially concerning road transport is still operated with very limited automated intelligence (Belle, Valckenaers, and Cattrysse 2012). Yard operations are responsible for processing vehicles, trailers and swap bodies outside the terminal building. They therefore have significant impact on the performance of internal sorting systems which can only be achieved by constantly high input flows and require minimized times for changing the transportation units for unloading and loading. Based on currently resulting effective maximum of sorting capacities, detailed analysis of yard operations and subsequent optimization is the main challenge to increase the performance of transshipment terminals.

As described in the following sections, a lot of different components are involved in yard operations in transshipment terminals. They interact in complex dependencies over time, corresponding to the characteristic arrival pattern which limits the possibility to apply static evaluation methods. Furthermore, given terminal layouts are the main focus of research because quick and flexible reaction on market requirements is needed and any new facilities can be build. Thus, the method of discrete event simulation is very suitable to analyze yard operations and management in logistic terminals. The method has been developed especially for container terminals and is used for case studies of real world scenarios as well as the analysis of controlling strategies and algorithms (Stahlbock and Voß 2008; de Koster, Le-Anh, and van der Meer 2004). Furthermore, it is necessary to develop simulation methods dedicated to terminals of road transportation such as cross docking facilities because they have to cope with individual challenges and characteristics (Rohrer 1995; de Koster, Le-Anh, and van der Meer 2004). It is stated that detailed modeling has to take place and actual delivery and shipping schedules have to form the system load. Additionally, it is important to include the yard activities and resources into the model (Rohrer 1995). Magableh et al. developed a model for a generic cross docking facility in a distribution network (Magableh, Rossetti, and Mason 2005). To easily change system loads it is based on statistical distributions for incoming shipments. The main focus is to analyze typical resource allocation problems within the terminal, all pro- 


\section{Clausen and Goedicke}

cesses are represented by average handling times and a fixed consolidation ratio is used. The approach of Liu and Takakuwa is dedicated to decision support in a retail cross docking facility (Liu and Takakuwa 2010). Implemented process chains are typical for retail distribution and the analysis is focused on operator and door utilization.

The existing methods do not focus on high system dynamics of transshipment terminals in open transport networks and do not take typical system loads of those logistics service providers into account. Tactical and operational decisions, such as door-destination-assignment or fork lift control are simplified in the models or are specifically dedicated to retail distribution. Furthermore, the approaches do not consider the complexity of yard operations, e.g. different vehicle types, actual travel distances and structural restrictions of the terminal building (e.g., loading heights). Therefore, the specific simulation suite "TransSim Node" based on Enterprise Dynamics has been developed in cooperation with Incontrol Simulation Solutions. The suite includes elements for yard operations as well as internal handling processes and deals with each vehicle and handling unit as individual objects (Neumann and Deymann 2008). It has been extended in this paper regarding different aspects to allow the application on real world scenarios. Therefore, section two describes yard operations and related management decisions in detail as well as the modeling approaches. In section 3 the method is applied on an exemplary terminal and different strategies are analyzed regarding their impact on performance aspects. The paper is summarized and concluded in section 4.

\section{MODELING OF YARD OPERATIONS AND YARD MANAGEMENT}

The main aspects of yard operations and management are described in sections 2.1 and 2.3, each followed by the description of the modeling.

\subsection{Yard Operations in Transshipment Terminals}

Shipments reach the first depot of the transport chain after their pickup at the shipping customer. Depending on the geographic structure of the region, shipment characteristics and customers different types of transportation units are used for pickup tours. Swap bodies or trailers are used by customers responsible for high amounts of shipments. The transport units are loaded directly at the customer's site and collected by a forwarder at a predefined time in the afternoon or evening. Customers may also consolidate shipments with the help of loading devices, such as pallets, box pallets or cages. In the afternoon small or medium weight-trucks with fixed body are executing pickup tours to collect the loading devices. This results in different types of trucks and transportation units arriving at the transshipment terminal in the afternoon and evening. Trailers and swap bodies have to be brought directly to the unloading gates or set down at specific areas on the yard. At the destination the supporting legs of the swap bodies have to be pulled out and fixed. For the trucks with fixed body the driving unit has to get unloaded as well. Furthermore, the different types of transportation units are characterized by different unloading heights at the gates. Concerning the unloading process itself, units directly loaded with shipments have to be differed from units with loading devices due to different technical equipment used at the gates. After the unloading process is finished, the transportation units without own propulsion system (trailers, swap bodies further referred to as 'unpowered units') are collected by the corresponding driving unit or have to be brought to buffer areas on the yard by special terminal trucks otherwise. The shipments get sorted by line haul destinations within the terminal and afterwards loaded at the loading gates. National line haul transports are usually operated by swap bodies which allow decoupled processes of loading the transport units and actually transporting them. Corresponding driving units and suitable trailers arrive at the yard shortly before predefined cut off times to collect the swap bodies which means that staff costs for the driver are mainly used for productive transportation processes. The shipments are loaded directly into the swap bodies.

The line haul transports arrive at the receiving depot during the night. Arrival times are determined by the geographic location of the shipping depot. If more than one truck is transporting the amount from one 


\section{Clausen and Goedicke}

depot to another, the first truck might arrive already quite early at the receiving depot. Regarding terminal operations in the inbound shift the trucks arrive at the terminal to deliver the swap bodies. As in the outbound shift they can be brought directly to the gates or set down on yard areas. Shipments are unloaded and sorted for the downstream delivery tours. As in the pickup process in the afternoon, different types of vehicles are used for deliveries due to the geographic distribution and shipment structure of the customers. In addition to swap bodies and medium weight trucks with fixed body, small delivery vans might be in use for home deliveries to end customers. For loading the shipments during inbound shift a lot of different gates for the delivery destinations are required. Regarding gate assignment, different loading heights of the used trucks have to be taken into account as well as technical loading equipment at specific gates or other business specific characteristics of delivery processes.

In addition to the described processes of the trucks, there are three main processes required for yard operations. Firstly, an essential aspect is the possibility to move around unpowered transportation units (further referred to as 'shunting'). Therefore, usually special terminal trucks are used which are advantageous to normal trucks regarding their maneuverability as well as the possibility to lift swap bodies very fast and transport them with pulled out supporting legs. Furthermore, two supporting processes have to be taken into account. Transporting on terminal roads is necessary to connect all destinations for trucks and transportation units on the yard. Additionally, waiting processes can occur for driving units due to high utilization of gates. Space is needed to buffer the trucks on the yard until loading or unloading can take place or the corresponding trailer is ready for picking up.

As a result of the described operating processes, different static and dynamic objects are necessary for yard operations in a transshipment terminal as they can be seen in figure 2. Static objects form the layout of the system which is the basis for the process chains of the dynamic objects that "move" through the system.

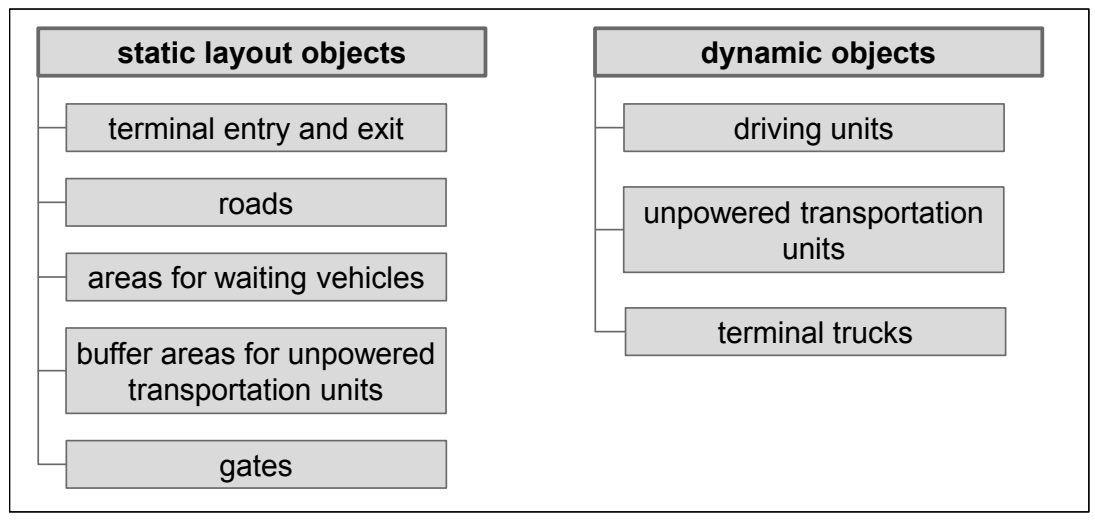

Figure 2: Objects of yard operations

\subsection{Modeling of Yard Objects}

The focus of modeling terminal yard operations as described above is to consider the essential characteristics of the involved objects and processes. When trucks arrive at the terminal site they have to pass a gatekeeper due to security reasons. Depending on local situations waiting areas might be established in front of the entry gate. Therefore, the terminal site's border is chosen as the system boundary for simulation. Different checking and assigning processes are executed at the gatekeeper which differ concerning truck types and operating shifts. By modeling the gatekeeper as an intelligent server (available in TransSim Node suite) and the corresponding waiting area as a queue, the essential characteristics are taken into account. Subsequent to the gatekeeper, roads are established on the yard which connect all possible destinations. For their modeling it is important to consider the actual distances between two specific locations as well as basic traffic rules at crossings. Therefore, a network of special road element objects is integrated into the yard models. Those objects are available in the suite TrafficSim of Enterprise Dynam- 


\section{Clausen and Goedicke}

ics and enable realistic movement of trucks based on the length of the element and the driving speed of the contained vehicle object. Terminals are modeled to scale to cover real distances.

Destinations for the vehicles are loading and unloading gates, areas for waiting vehicles as well as areas for buffering unpowered transportation units. It is essential to model each of them as single items with realistic dimensions and positions on the yard to take the actual availability of free space on the yard into account. Therefore, elements for waiting vehicles and buffered unpowered transportation units are used from the TransSim Node suite. Driving units can move on those objects because they are derived from the road element. Furthermore, additional process times at the area are considered (e.g., positioning of trailer on the buffer area and decoupling). Those modeling aspects also apply for the gate object of TransSim Node. Due to the fact that the gate is the interface between the external yard processes and the internal handlings, the ramp is modeled including its technical parameters (e.g. time for gate opening).

Trucks in TransSim Node are created by a vehicle generator in correspondence to an input table which defines single vehicles including all parameters and arrival times (Neumann and Deymann 2008). It has to be distinguished between different truck types and transportation units. Therefore, components of a vehicle are modeled and combined to build a specific truck type. A vehicle consists of a driving unit which is the powered part needed to move around. Additionally, devices to load swap bodies and swap bodies themselves can be necessary as well as trailers or semi-trailers. All are unpowered and have to be combined with a driving unit to get to a destination. Tables define the possible combinations of components which are the basis for vehicle creation in the vehicle generator. This modular approach also allows modeling of new combinations such as 60-ton trucks, for example. An additional attribute defines the state of the transportation units (full, empty). The status of a vehicle is coded by a vehicle index which describes the exact configuration, such as 'driving unit with empty swap body and trailer with empty swap body', for example. Accordingly, the status changes by the movement of the truck on the yard (changing into 'driving unit with empty swap body and empty trailer' after set down of empty swap body on trailer). All vehicle configurations relevant for logistics service providers can be modeled used for the simulation study.

Besides this detailed modeling of different truck types from a technical point of view, the logistics characteristics of a truck have to be considered to allow realistic yard operations. As described above and in the following section, distinctions have to be made especially regarding assignment of transportation units to gates and buffer areas. Therefore, a numerical system to easily distinguish the incoming vehicles has been established that informs about the logistics characteristics and does not change during the movement through the system:

- $\quad$ involved in which operation shift

- $\quad$ type of loading (directly, loading devices, mixed)

- fixed body truck or swap body truck

- $\quad$ with or without trailer

The resulting indicator is assigned to each component of an incoming vehicle and can be used for differentiated queries within the simulation procedures.

The dynamic objects of internal terminal activities are shipments and loading devices. They leave the transportation unit at unloading gates which means that they are created as individual items at the gate. Subsequently, they go through unloading, sorting and loading processes which are modeled in an aggregated way because yard processes are the focus of analysis.

\subsection{Yard Management Decisions}

To manage the dynamic objects, a central control is established in most cases where experienced personnel is doing the general management of internal as well as yard operations with the help of surveillance cameras and supporting software tools. Typically, those tools administrate the current status of yard resources, for example if a specific yard area is in use or not. This information is used by the operations manager to assign destinations to trucks and shunting tasks to terminal trucks according to his experience. 


\section{Clausen and Goedicke}

Three main events have to be coordinated for management of yard operations (idea according to Neumann and Szewczyk 2008). The first is at the gate of a terminal. Vehicles arriving at the yard have to be assigned to a destination according to predefined processes which depend on load type, truck type and operating shift. Furthermore, the current utilization of the different area types has to be taken into account. Secondly, when the truck ends the task at the defined destination the following process has to be coordinated. It has to be distinguished between different area types, the current task of the driving unit and the transportation unit's characteristics. If an unpowered unit is involved in the event, even two decisions are necessary. For example, if the driving unit dropped of a trailer at a gate, the destination for the driving unit has to be determined in this step. Additionally, loading or unloading for the trailer has to be initiated. In some systems the assignment for both units, driving unit and trailer, already takes place at the gatekeeper. The third decision event occurs at the gates when the loading or unloading process has been finished. It has to be determined how the next planned process can be realized. If, as an example, the driving unit's task is to collect its trailer the status and current location of the trailer has to be determined and matched with the request of the driving unit. When the trailer has not finished its loading or unloading process yet the driving unit has to be assigned to a waiting area. Otherwise, the current location of the trailer is assigned to the driving unit as its next destination.

If an unpowered unit is involved in an event of the second or third type, an additional yard management task can occur. Due to the fact that the unit cannot move to the assigned destination itself it has to be determined if a terminal truck is necessary or a corresponding driving unit can perform this task. If a terminal truck is needed the task has to be created and assigned to a terminal truck. Usually, the task with longest waiting time is assigned to the first free terminal truck. Sometimes individual priorities are allocated to specific tasks by the operations manager. It is also possible that terminal truck drivers can see all waiting tasks and decide about the next task on their own. Further detailed information is often missing, e.g. on the loading characteristics of transportation units, as well as decision support, e.g. by standardized rules for assignment. Concluding, the efficiency of yard operations is largely depending on skills and experience of the operations managers.

\subsection{Modeling of Yard Management}

The central control unit in models created with the help of TransSim Node is the 'Yard Management' (description of basic modeling based on Neumann and Szewczyk 2008). This important component coordinates any vehicle movement in the yard. In order to manage those complex and dynamic processes, including constantly altering circumstances, the current state of the system needs to be analyzed permanently. Therefore, the Yard Management gets information about recent events in the model as well as routing requests of single vehicles from the other components of the model. According to this information the Yard Management refreshes system data and decides on further actions of the respective vehicle.

Data is monitored with the help of tables, containing information on characteristics of static and dynamic objects as well as on their current status. Tables can be differentiated into four types. Overview tables contain general information about vehicles and trailers, consignments and destinations. Input tables define the input data of the system. They include times of arrival of vehicles and the assignments of consignments to vehicles and destinations as well as detailed characteristics of each involved vehicle and consignment.

The third group are static tables which are the basis for vehicle routing on the yard. Activity chains for common vehicle types have been developed and coded in the tables, which define the vehicle's process chain depending on the vehicle configuration and activity category (loading, unloading or both). Based on different types of incoming vehicles, travel chains are defined which correspond to the tasks truck drivers are typically executing on the terminal yard. Since several haulage companies drive for a logistic service provider, those tasks are defined in advance, for example if a truck that distributes a loaded swap body to the terminal, leaves the terminal directly or loads a swap body again. The travel chains are serially numbered and for each process the actual task (e.g., decoupling a trailer), the required area (e.g., 


\section{Clausen and Goedicke}

buffer area for unpowered units) and the resulting vehicle index after the task has been executed are defined. It is decided dynamically within the simulation run if a task in the chain can already be executed or if it still necessary to execute (e.g., if a picked up trailer has to be loaded before leaving the terminal or has already been loaded). Based on this logic of TransSim Node activity chains have been modeled which are relevant for simulation of yard operations in real terminals. Additionally, it was necessary to implement new tasks into the framework, such as decoupling a trailer with a swap body on it, for example.

The last group of tables are dynamic tables. They are used to manage the current states of yard objects as well as shunting and assignment of unpowered transportation units. For each type of static yard objects, excluding roads, a table is generated which includes characteristics, for example if a gate is used for loading or unloading, as well as the current status which is dynamically adjusted. Furthermore, shunting tasks are managed in an additional table to control each task starting with the request of an unpowered unit to get moved until the task is successfully completed. Additionally, a table controls the assignment of unpowered units to driving units. Every event in the system as described in chapter 2.3 initiates a refreshing of the data. Due to consistency of programming all events have to be passed through.

To control the specific processes of transshipment terminals further implementations have been made for the paper. An additional table has been integrated to manage the assignment of swap bodies to loading doors according to relevant destinations and shipment volumes which is necessary because swap bodies are basically independent from a specific vehicle and loading information has to be known in advance to truck arrival. Based on the numerical system to distinguish incoming vehicles the logic of assigning gates and yard areas has been detailed to consider different strategies in the operation shifts. Additionally, the controlling of terminal trucks has been enhanced. At first, commonly used strategies as assignment by longest task waiting time have been implemented. Furthermore, a possibility for flexible consideration of different priorities for task characteristics has been developed. By individual implementation specific rules have been realized, as for example the assignment of two gates for driving unit and trailer at the gatekeeper.

\section{EXEMPLARY EXPERIMENTS AND RESULTS}

The suite for modeling yard operations and management is applied on a real terminal of a logistics service provider. Due to predicted shipment volumes the first aim of the study is measuring the system performance using future system loads taking all dependencies into account, especially concerning yard operations. The second aim is to evaluate the impact of different operating strategies on system performance aspects.

As preparatory work the layout of the system has been mapped to build each single component of the facility to scale within the simulation model. Existing layout generating tools (Clausen et al. 2011) have been used and adapted for yard components to support model building. Additionally, the processes have been mapped and transferred into event-driven process chains as a basis for determining the relevant activity chains for the different vehicle types and scenarios. Process times for handling unpowered units have been determined in the real system and implemented into the models (decoupling of trailer, setting down of swap body from normal driving units, setting down swap body from terminal truck etc.). Subsequently, system load data has been prepared and simulation experiments have then been executed and analyzed. To guarantee a holistic view on system performance aspects, different information is gathered within the simulation runs: the movement of each vehicle through the system is documented (time, place) as well as all information on terminal truck tasks and performance of internal sorting. Intelligent evaluation of this data results in throughput times of vehicles, which can be divided into time at a gate, time for waiting on a free gate and yard processing time which includes all driving and shunting processes on the yard. Furthermore, resource utilization for external areas can be derived from the monitored data as well as different performance indicators for terminal trucks, such as the average execution time of a shunting task and the average waiting time of a task for a free terminal truck. To complete the holistic view on system performance, the efficiency of internal resources has to be measured. As the key figure, the unloading performance is analyzed because it determines which shipment volume is actually supplied from the yard 


\section{Clausen and Goedicke}

to the terminal. The performance is calculated as the ratio of the amount of shipments being unloaded per operating shift and the time unloading gates have been used in total (shipments per hour).

\subsection{Scenario Analysis and Comparison of Results}

Three different scenarios are analyzed in the following paragraphs. Starting with the current situation of yard operations, swap body handling is changed in the second scenario to enhance handling performance of the terminal. Due to the fact that this strategy change initiates additional work load for shunting, the third scenario enhances the control of terminal trucks.

For the basic scenario the current situation regarding activity chains for vehicles is applied. Vehicles deliver the swap bodies directly to the gates. After the first swap body is set down, the empty trailer is brought to a buffer area. Subsequently, the second swap body is set down at a gate and finally the empty trailer is fetched again before the truck leaves the facility. Terminal trucks are assigned to the task with longest waiting time. Verification and validation has been done by trace-analysis of single objects and monitoring of basic performance indicators in the first step.

As a result, throughput times for the different vehicle types are determined. Yard processing times occur for all vehicles and account for 13 to 33 minutes of throughput time. Waiting times are relevant for trucks with fixed bodies which actually have to wait for a free gate. Also trucks picking up swap bodies may have waiting times because loading has not been completed at the time of truck arrival. Results show, for example, that waiting times increase up to 59 minutes for the last arriving fixed body trucks at the end of the outbound shift due to limited unloading capacity. This shows the relations between transport and terminal operations and supports the need of cooperative planning processes. Analysis of terminal trucks shows that average execution time of a task is 3.9 minutes. The results are compared to evaluations of the real system and have also been validated in cooperation with experts operating the real terminal.

In the second scenario the strategy for swap body handling within the terminal is changed. Vehicles bringing swap bodies for unloading are setting them down in yard areas. They are brought to the gates only by terminal trucks. On the one hand, this enables higher utilization of gates due to the fact that the time consuming process of pulling out the supporting legs of the swap bodies does take place in advance. On the other hand, the workload for terminal trucks is supposed to increase because all swap bodies have to be handled by terminal trucks. To model this scenario, the activity chains are changed for all vehicles bringing swap bodies into the terminal. Furthermore, special buffer areas are implemented which allow setting down of two swap bodies without decoupling of the trailer.

Results show that the throughput time for vehicles bringing swap bodies can be reduced significantly by over $30 \%$ for local pickup transports. In the inbound shift swap bodies are exclusively used and a certain amount is available on the yard before operations actually start. Therefore, throughput times can only be reduced by $3 \%$. Additionally, the unloading performance can be increased by $5 \%$ in outbound and $1.9 \%$ in inbound operations. In contrast to that, overall operating efforts of terminal trucks grow by $10 \%$ and the process time for executing a single shunting task increases to 4 minutes in average. Furthermore, the average waiting time for a free terminal truck rises by $23 \%$.

Based on the results of the second scenario, the third scenario tries to improve the performance of terminal trucks. The dispatching strategy is changed and considers the origin of the shunting task in addition to the waiting time. Tasks with a short distance between the pickup point of the unpowered transportation unit and the current location of the involved terminal truck are prioritized. If more than one task is in the same area as the terminal truck and can be assigned, the one with longest waiting time is chosen. This has been identified as a good basic dispatching rule to improve utilization of vehicle systems (e.g., Klein and Kim 1996; Goel and Gruhn 2008). The processes of vehicles remain as in the second scenario, thus their throughput times will not change. Average executing time of shunting tasks can be reduced to 3.8 minutes and the average waiting time of shunting tasks for a free terminal truck decreases by $36 \%$. In contrast to that, indicators for unloading performance get worse for inbound operations by $2.2 \%$. However, for the outbound shift unloading performance increases slightly by almost $1 \%$. This illustrates that 


\section{Clausen and Goedicke}

strategies do not result in the same impact on performance for all operating shifts as different mixes of vehicles are used and the areas on the yard are used in a different way.

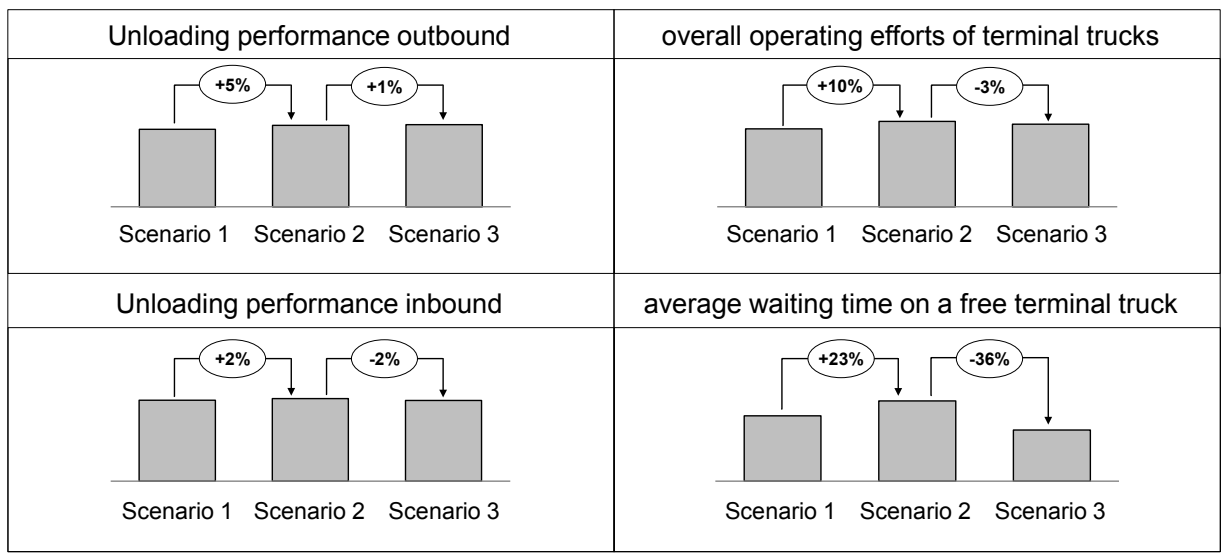

Figure 3: Comparison of results

The comparison of scenarios as illustrated in figure 3 shows the high impact different yard management strategies have on performance aspects of the whole terminal. A strategy improving the unloading performance in scenario 2 significantly increases the shunting effort. However, by improving the efficiency of terminal truck operations in scenario 3, the unloading performance is decreased again in the inbound shift.

\section{CONCLUSIONS}

The paper firstly describes the high impact of yard operations and management on the performance of transshipment terminals and hence the competitiveness of logistics service providers. Furthermore, it shows the complex dependencies of the different involved components over time which limit the applicability of static evaluation methods. Therefore, a discrete-event simulation approach is developed to allow holistic analysis of yard management and operations. It is applied on a real transshipment terminal and the ability to implement and analyze different strategies is shown. Furthermore, the comparison of three scenarios illustrates the conflict between opportunities to improve resource utilization on the one hand and increase terminal performance on the other hand. Concluding, additional research has to be done on the determination of best strategy combinations for yard management decisions regarding various system loads which maximize handling performance at minimal use of resource. Those results could be integrated into terminal management applications and support the respective personnel by enhancing performance and managing increasing shipment volumes.

\section{ACKNOWLEDGMENTS}

We thank the NRW Graduate School of Energy Efficient Production and Logistics for funding the research on simulation of yard operations and management in transshipment terminals.

\section{REFERENCES}

Belle, J.V., P. Valckenaers, and D. Cattrysse. 2012. "Cross-docking: State of the art" Omega - the international journal of management science 40:827-846.

Clausen, U., I. Goedicke, L. Mest, and J. Kaffka. 2011. "Layout Planning of Less Than Truckload Terminals" In Proceedings of the 9th Industrial Simulation Conference 2011, Edited by S. Balsamo, A. Marin, 204-208. 
de Koster, R., T. Le-Anh, and J.R. van der Meer. 2004. "Testing and classifying vehicle dispatching rules in three real-world settings" Journal of Operations Management 22:369-386

Goel, A., and V. Gruhn. 2008. "A General Vehicle Routing Problem" European Journal of Operations Research 191:650-660.

Klein, C., and J. Kim. 1996. "AGV Dispatching” International Journal of Production Research 34:95110.

Liu, Y., and S. Takakuwa. 2010. "Enhancing simulation as a decision-making support tool for a CrossDocking Center in a Dynamic Retail-Distribution Environment" In Proceedings of the 2010 Winter Simulation Conference, Edited by B. Johansson, S. Jain, J. Montoya-Torres, J. Hugan, and E. Yücesan, 2089-2100. Piscataway, New Jersey: Institute of Electrical and Electronics Engineers, Inc.

Magableh, G. M., M. Rossetti, and S. Mason. 2005. "Modeling and Analysis of a Generic Cross-Docking Facility" In Proceedings of the 2005 Winter Simulation Conference, Edited by M. E. Kuhl, N. M. Steiger, F. B. Armstrong, and J. A. Joines, 1613-1620. Piscataway, New Jersey: Institute of Electrical and Electronics Engineers, Inc.

Neumann, L., and S. Deymann. 2008. "TRANSSIM-NODE - a simulation tool for logistics nodes" In Proceedings of the 6th Industrial Simulation Conference 2008, 283-287. Lyon.

Neumann, L., and M. Szewczyk. 2008. "Abbildung von Yard-Management-Prozessen in Simulationsmodellen" In Advances in Simulation for Production and Logistics Application, 289-298. Stuttgart, Fraunhofer IRB Verlag.

Rohrer, M. 1995. "Simulation and Cross Docking" In Proceedings of the 1995 Winter Simulation Conference, Edited by C. Alexopoulos, K. Kang, W. R. Lilegdon, and D. Goldsman, 846-849. Piscataway, New Jersey: Institute of Electrical and Electronics Engineers, Inc.

Stahlbock, R., and S. Voß. 2008. "Operations Research at Container Terminals: a Literature Update" OR Spectrum 30:1-52

\section{AUTHOR BIOGRAPHIES}

UWE CLAUSEN is the managing director of the Institute of Transport Logistics at TU Dortmund University and director of the Fraunhofer-Institute for Material Flow and Logistics (IML). He studied computer sciences at the University of Karlsruhe $(\mathrm{TH})$ and finished his doctoral thesis on transportation network optimization at TU Dortmund University in 1995. Afterwards, he worked at the "Deutsche Post AG" and joined Amazon.de in 1999. In 2001 Uwe Clausen accepted the chair "Transport Systems and Logistics" at TU Dortmund University, which became the Institute of Transport Logistics in 2011. He is amongst others chairman of EffizienzCluster LogistikRuhr and a member of ECTRI European Conference of Transport Research Institutes and of the Scientific Advisory Board of German Logistics Association (BVL).

INA GOEDICKE is a Ph.D. student at the Institute of Transport Logistics. She studied Logistics at TU Dortmund University and obtained her degree in 2009. She does research in the fields of logistic terminal layout, yard management strategies and simulation in traffic and transport logistics. 